\title{
Repercussões Hemodinâmicas do Posicionamento em Litotomia Exagerada para Histerectomia Vaginal em Paciente Cardiopata. Relato de Caso *
}

\section{Hemodynamic Repercussions of Exaggerated Lithotomy Position for Vaginal Hysterectomy in Cardiac Patient. Case Report}

Roberto Cardoso Bessa Junior, TSA ${ }^{1}$; Agnaldo L. Silva Filho ${ }^{2}$; Plínio V. Maia ${ }^{3}$; Lúcio O. Quites ${ }^{4}$; Sérgio A. Triginelli ${ }^{5}$

\begin{abstract}
RESUMO
Bessa Jr RC, Silva Filho AL, Maia PV, Quites LO, Triginelli AS Repercussões Hemodinâmicas do Posicionamento em Litotomia Exagerada para Histerectomia Vaginal em Paciente Cardiopata. Relato de Caso
\end{abstract}

JUSTIFICATIVA E OBJETIVOS: A técnica de histerectomia vaginal possibilita menor tempo operatório e o uso do bloqueio do neuro-eixo, com os benefícios de melhor analgesia pós-operatória e menor resposta sistêmica ao procedimento cirúrgico. O objetivo deste relato foi descrever as alterações hemodinâmicas decorrentes do posicionamento em litotomia exagerada em paciente cardiopata.

RELATO DO CASO: Paciente de 33 anos, GOPOAO, com história de sangramento uterino anormal e anemia. A ultra-sonografia evidenciava útero miomatoso com volume estimado de $420 \mathrm{~cm}^{3}$. Ela era portadora de trombofilia e miocardiopatia dilatada, com passado de dois acidentes vasculares encefálicos isquêmicos e dois infartos agudos do miocárdio. Foi monitorizada com pressão arterial invasiva e cateter de artéria pulmonar com medida de débito cardíaco contínuo. Realizada raquianestesia com bupivacaína hiperbárica e morfina. A paciente foi posicionada em litotomia exagerada sendo realizada histerectomia total pela técnica de Heaney e salpingectomia bilateral. Como intercorrência intra-operatória apresentou diminuição do índice cardíaco e aumento das pressões de câmaras direitas após o posicionamento, necessitando do uso de dobutamina.

CONCLUSÕES: O posicionamento em litotomia exagerada pode ocasionar alterações hemodinâmicas que devem ser consideradas na escolha da técnica cirúrgica.

* Recebido do (Received from) Hospital das Clínicas da Universidade Federal de Minas Gerais, Belo Horizonte, MG

1. Especialista em Medicina Intensiva. Anestesiologista do HC/UFMG e Hospital Lifecenter

2. Especialista em Cirurgia Geral, Ginecologia e Obstetrícia. Doutor em Ginecologia pela UNESP

3. $\mathrm{ME}_{3}$ do CET do Hospital das Clínicas da UFMG

4. Especialista em Anestesiologia. Professor Assistente da Faculdade de Medicina da UFMG

5. Especialista em Ginecologia e Obstetrícia. Professor Adjunto da Faculdade de Medicina da UFMG

Apresentado (Submitted) em 18 de maio de 2005

Aceito (Accepted) para publicação em 31 de outubro de 2005

Endereço para correspondência (Correspondence to)

Dr. Roberto Cardoso Bessa Júnior

Rua Itamonte, 35/602 - Bairro Floresta

31110-220 Belo Horizonte, MG

E-mail: robertocarolina@uol.com.br

(C) Sociedade Brasileira de Anestesiologia, 2006
Unitermos: CIRURGIA, Ginecológica: histerectomia vaginal;

DOENÇAS, Cardíaca; POSIÇÃO: litotomia exagerada;

TÉCNICAS ANESTÉSICAS, Regional: subaracnóidea

\section{SUMMARY}

Bessa Jr RC, Silva Filho AL, Maia PV, Quites LO, Triginelli AS Hemodynamic Repercussions of Exaggerated Lithotomy Position for Vaginal Hysterectomy in Cardiac Patient. Case Report

BACKGROUND AND OBJECTIVES: Vaginal hysterectomy shortens surgery duration and may be performed with neuraxial block, which promotes better postoperative analgesia and lower systemic response to surgical procedure. This report aimed at describing hemodynamic changes promoted by exaggerated lithotomy position in cardiac patient.

CASE REPORT: Female patient, 33 years old, with history of abnormal uterine bleeding and anemia. Ultrasound revealed myomas of approximately $420 \mathrm{~cm}^{3}$. Patient had thrombophilia and dilated cardiomyopathy, with history of two ischemic strokes and two acute myocardial infarction. Monitoring consisted of invasive blood pressure and pulmonary artery catheter for continuous cardiac output measurement. Spinal anesthesia was performed with hyperbaric bupivacaine and morphine. Patient was placed in exaggerated lithotomy position being total hysterectomy performed by the Heaney technique and bilateral salpingectomy. Intraoperative intercurrences were post-positioning decreased cardiac output and increased right chambers pressure requiring dobutamine. CONCLUSIONS: Exaggerated lithotomy position may promote hemodynamic changes which should be considered when choosing the surgical technique.

Key Words: ANESTHETIC TECHNIQUES, Regional: spinal block; DISEASES, Cardiac; POSITION: exaggerated lithotomy; SURGERY, Gynecological, vaginal hysterectomy

\section{INTRODUÇÃO}

A s doenças cardiovasculares são as alterações clínicas mais freqüentemente encontradas na prática anestésica e representam causas importantes de morbimortalidade peri-operatória ${ }^{1}$. As repercussões do ato anestésico-cirúrgico implicam em sobrecarga adicional ao sistema cardiovascular, exigindo um aumento na demanda cardíaca, que pode ser facilmente tolerada por pacientes normais, mas que resultam em complicações significativas nos pacientes com doença cardíaca ${ }^{2}$. A resposta adrenérgica à estimulação cirúrgica associada aos efeitos circulatórios dos agentes anestésicos, da intubação traqueal, da ventilação com pressão positiva, das perdas sanguíneas, dos deslocamentos de líquido, do posicionamento cirúrgico, da dor pós-operatória, é alguns desses fatores. Eventos pós-operatórios 
relacionados ao óbito incluem o infarto agudo do miocárdio (IAM), as disritmias cardíacas e a insuficiência de múltiplos órgãos, secundária ao baixo débito cardíaco ${ }^{2}$.

A técnica de histerectomia vaginal, descrita por Heaney em 1934, apresenta algumas vantagens em relação à via abdominal. A via vaginal proporciona uma menor manipulação de alças intestinais, um pós-operatório menos doloroso, além de um menor tempo de internação e convalescença ${ }^{3-6}$. No entanto, para a realização da histerectomia por essa via é imprescindível o posicionamento da paciente em litotomia exagerada que pode se relacionar a repercussões hemodinâmicas significativas ${ }^{7}$. O presente relato descreveu o caso de uma paciente com miocardiopatia dilatada, submetida a histerectomia via vaginal, associada a monitorização hemodinâmica invasiva e a bloqueio de neuro-eixo.

\section{RELATO DO CASO}

Paciente com 33 anos, negra, obesa (índice de massa corporal: 32), GOPOA0 com história de sangramento uterino anormal com repercussão nos níveis hematimétricos (hemoglobina $5,7 \mathrm{~g} / \mathrm{dL}$ ), em melhora com o tratamento clínico. O exame pélvico mostrava boa amplitude vaginal e útero aumentado de volume com a mobilidade preservada. A ultra-sonografia evidenciava útero miomatoso com volume estimado de $420 \mathrm{~cm}^{3}$.

Era portadora de trombofilia de etiologia não esclarecida, com passado de dois acidentes vasculares encefálicos sem seqüelas e história de um IAM anterior extenso havia oito anos com evolução em choque cardiogênico e um IAM sem supra desnivelamento do segmento ST havia um ano. O ecocardiograma mostrava aumento do átrio $(50 \mathrm{~mm})$ e do ventrículo $(67 \mathrm{~mm}$ ) esquerdos, aumento das câmaras direitas, acinesia nas paredes septal, posterior, inferior e ápex, hipocinesia acentuada das paredes anterior e lateral; sendo a fração de ejeção estimada de 0,28 . A cineangiocoronariografia mostrava uma circulação coronariana com padrão de domi- nância direita, artérias coronárias sem lesões significativas e hipocinesia difusa grave do ventrículo esquerdo. Devido à ausência de aterosclerose, a síndrome coronariana foi atribuída a fenômenos trombóticos secundários a trombofilia. Encontrava-se em uso domiciliar de enalapril ( $5 \mathrm{mg}$ a cada 12 horas), espironolactona ( $25 \mathrm{mg}$ ao dia), furosemida (40 mg ao dia), carvedilol ( $25 \mathrm{mg}$ a cada 12 horas) e warfarin ( $7 \mathrm{mg}$ ao dia).

Quatro dias antes da cirurgia foi suspenso o warfarin e, iniciada a administração de enoxaparina ( $80 \mathrm{mg}$ ) a cada 12 horas. A última dose de enoxaparina foi administrada 24 horas antes da cirurgia, com INR e TTPa dentro dos valores de referência na véspera da cirurgia. Aenoxaparina foi reiniciada 12 horas após o término da cirurgia e o warfarin, no dia seguinte. As medicações de uso crônico foram mantidas. Amedicação pré-anestésica consistiu de lorazepan $(2 \mathrm{mg})$ às 22 horas na véspera da cirurgia e às 6 horas na manhã da cirurgia com jejum a partir das 24 horas.

Na sala cirúrgica a paciente foi monitorizada de forma não-invasiva e, após sedação, sob anestesia local foi introduzido cateter na artéria radial esquerda e inserido um cateter de artéria pulmonar (CAP) através da veia subclávia direita, sendo utilizado um monitor de débito cardíaco contínuo (Baxter-Edwards ${ }^{\circledR}$ ). Após a monitorização foi realizada a medida dos parâmetros hemodinâmicos basais. A seguir, feita a raquianestesia com administração de $15 \mathrm{mg}$ de bupivacaína hiperbárica a 0,5\% e $200 \mu \mathrm{g}$ de morfina. Cerca de 15 minutos após a punção lombar, com o nível sensitivo em $\mathrm{T}_{8}$, a paciente foi colocada em posição de litotomia exagerada para a realização da histerectomia por via vaginal pela técnica de Heaney com salpingectomia bilateral. Durante a cirurgia foram administrados $4 \mathrm{mg}$ de ondansetron e $8 \mathrm{mg}$ de dexametasona, para a profilaxia de náuseas e vômitos pós-operatórios, e $1200 \mathrm{~mL}$ de solução fisiológica a 0,9\%. Os parâmetros hemodinâmicos foram registrados antes do bloqueio, 20 minutos após o bloqueio, após o início da dobutamina por bomba de infusão, 5 minutos após a retirada da paciente da posição de litotomia exagerada e 20 minutos de-

Tabela I - Parâmetros Hemodinâmicos Peri-Operatórios

\begin{tabular}{|c|c|c|c|c|c|}
\hline & $\begin{array}{c}\text { Antes da } \\
\text { Raquianestesia } \\
\end{array}$ & $\begin{array}{c}\text { Cinco Minutos após o } \\
\text { Posicionamento }\end{array}$ & $\begin{array}{c}\text { Com } \\
\text { Dobutamina } \\
\end{array}$ & $\begin{array}{c}\text { Após a Retirada da Posição } \\
\text { de Litotomia Forçada }\end{array}$ & $\begin{array}{l}\text { Após } 20 \text { Minutos da } \\
\text { Retirada da Posição }\end{array}$ \\
\hline PAM & $85 \mathrm{mmHg}$ & $94 \mathrm{mmHg}$ & $110 \mathrm{mmHg}$ & $104 \mathrm{mmHg}$ & $104 \mathrm{mmHg}$ \\
\hline PVC & $3 \mathrm{mmHg}$ & $9 \mathrm{mmHg}$ & $10 \mathrm{mmHg}$ & $8 \mathrm{mmHg}$ & $8 \mathrm{mmHg}$ \\
\hline PAPm & $31 \mathrm{mmHg}$ & $28 \mathrm{mmHg}$ & $40 \mathrm{mmHg}$ & $28 \mathrm{mmHg}$ & $36 \mathrm{mmHg}$ \\
\hline PCP & $15 \mathrm{mmHg}$ & $22 \mathrm{mmHg}$ & $28 \mathrm{mmHg}$ & $15 \mathrm{mmHg}$ & $23 \mathrm{mmHg}$ \\
\hline $\mathrm{FC}$ & 83 bpm & 75 bpm & 70 bpm & $70 \mathrm{bpm}$ & 75 bpm \\
\hline DC & 5,4 & 4,8 & 6 & 6,1 & 5,6 \\
\hline IC & 2,7 & 2,4 & 3 & 3,1 & 2,8 \\
\hline IRVS & 2423 & 2845 & 2654 & 2499 & 2718 \\
\hline IRVP & 473 & 201 & 319 & 338 & 368 \\
\hline IVS & 33 & 32 & 43 & 44 & 38 \\
\hline
\end{tabular}

PAM - pressão arterial média ( $\mathrm{mmHg}$ ), PVC - pressão venosa central (mmHg), PAPm - pressão média de artéria pulmonar (mmHg), PCP - pressão capilar pulmonar ( $\mathrm{mmHg})$, FC - freqüência cardíaca, DC - débito cardíaco (L/min), IC - índice cardíaco, IRVS - índice de resistência vascular sistêmica, IRVP - índice de resistência vascular pulmonar, IVS - índice de volume sistólico 
pois do término da cirurgia (Tabela I). Como intercorrência intra-operatória apresentou diminuição do índice cardíaco e aumento das pressões de câmaras direitas após o posicionamento. Acirurgia transcorreu em 35 minutos e o procedimento anestésico em $1 \mathrm{~h} 50$ minutos; com o término do procedimento a paciente foi encaminhada ao CTI onde permaneceu por 24 horas. Recebeu alta hospitalar no terceiro dia de pós-operatório.

\section{DISCUSSÃO}

A avaliação pré-operatória de um cardiopata deve levar em conta os fatores clínicos do paciente, os fatores relacionados à cirurgia e a sua capacidade funcional.

O objetivo dessa estratificação de risco cardíaco pré-operatório é propiciar mudanças no manuseio peri-operatório com intuito de diminuir a morbimortalidade. Pode-se adiar a cirurgia devido a sintomas instáveis, realizar um preparo pré-operatório específico (estabilização farmacológica, volêmica ou mecânica), realizar intervenções pré-operatórias (valvoplastia, implante de marcapasso, angioplastia, cirurgia de revascularização miocárdica), optar por mudanças na monitorização intra-operatória (monitorização hemodinâmica invasiva, ecocardiograma transesofágico), e definir o pós-operatório em unidades de terapia intensiva ${ }^{8}$. A paciente em questão encontrava-se compensada do ponto de vista cardiovascular, encontrando-se na classe funcional III da New York Heart Association (NYHA), o que a colocava com preditores clínicos de maior gravidade e que seria submetida a uma intervenção cirúrgica de risco intermediário.

A monitorização hemodinâmica invasiva foi realizada com cateter de PIAe CAP (pressão intra- arterial e cateter de artéria pulmonar), sendo a paciente encaminhada para a unidade de terapia intensiva no pós-operatório. O uso da monitorização descrita possibilitou o manuseio volêmico intra-operatório, o uso de dobutamina e o diagnóstico dos efeitos hemodinâmicos do posicionamento. Na monitorização inicial encontrava-se com PVC de $3 \mathrm{mmHg}$ e PCP de $16 \mathrm{mmHg}$. A administração de $500 \mathrm{~mL}$ de solução fisiológica a $0,9 \%$ como teste volêmico elevou a PVC para 6 e a PCP para $22 \mathrm{mmHg}$, demonstrando uma insuficiência ventricular esquerda grave por restrição diastólica.

Optou-se pela histerectomia vaginal devido a seu tempo cirúrgico menor e a sua menor resposta inflamatória pós-operatória, quando comparada à via abdominal ${ }^{4-6}$. Mesmo em pacientes com úteros de peso superior a 250 gramas a via vaginal apresenta menor tempo de internação ${ }^{1,9}$ versus 3,7 dias; $p<0,001)$ e íleo pós-operatório $(1,1 \%$ versus $10,5 \% ; p=$ 0,006 ) em relação à via abdominal, sem aumento da morbidade ${ }^{6}$.

A posição cirúrgica utilizada foi a litotomia exagerada com céfalo-declive. Esta posição combina as piores características de ambas posições isoladas. Na posição de litotomia exagerada há a compressão das vísceras abdominais com subseqüente elevação do diafragma e compressão pulmonar (levando ao aumento das pressões intratorácicas e à alteração na relação ventilação-perfusão) e redução do retor- no venoso. Na posição de céfalo-declive há o aumento do consumo de oxigênio pelo miocárdio, aumento da pressão intracraniana e vasodilatação reflexa ${ }^{7}$. Constituem riscos associados ao posicionamento cirúrgico à síndrome do compartimento abdominal, rabdomiólise, neuropatias, disjunção da sínfise púbica, isquemia de membros inferiores, úlceras e alopécia de pressão, além da hipotensão arterial $^{7,9,10}$. Às vezes, pela baixa tolerância à posição, é necessário o controle da ventilação. No presente caso após o posicionamento em litotomia exagerada foi diagnosticado pelas medidas hemodinâmicas: diminuição do índice cardíaco, aumento na resistência vascular sistêmica, elevação da pressão de artéria pulmonar e de capilar pulmonar. Foi iniciada dobutamina na dose de $5 \mu \mathrm{g} \cdot \mathrm{kg}^{-1} \cdot \mathrm{min}^{-1}$ no intuito de melhorar o índice cardíaco e reduzir as pressões de artéria pulmonar, capilar pulmonar e resistência vascular sistêmica. O efeito deletério do posicionamento em uma paciente com grave comprometimento cardíaco foi comprovado pela melhora das medidas hemodinâmicas após o retorno ao decúbito dorsal com redução na infusão de dobutamina e sua posterior suspensão.

A combinação de técnicas anestésico-cirúrgicas adequadas permite maior segurança e diminuição da morbimortalidade em pacientes críticos. A técnica de histerectomia vaginal é segura em pacientes com cardiopatia grave, no entanto, apresenta alterações hemodinâmicas decorrentes do posicionamento em litotomia exagerada que podem ser mal toleradas e acarretar graves complicações cardiovasculares.

\section{Hemodynamic Repercussions of Exaggerated Lithotomy Position for Vaginal Hysterectomy in Cardiac Patient. Case Report}

Roberto Cardoso Bessa Junior, TSA, M.D.; Agnaldo L. Silva Filho, M.D.; Plínio V. Maia, M.D.; Lúcio O. Quites, M.D.; Sérgio A. Triginelli, M.D.

\section{INTRODUCTION}

Cardiovascular diseases are the most frequent clinical changes in the anesthetic practice and are major perioperative morbidity/mortality factors ${ }^{1}$. Anesthetic/surgical procedure repercussions imply additional cardiovascular overload, requiring increased cardiac demand which may be easily tolerated by normal patients, but which result in significant complications for cardiac patients ${ }^{2}$. Adrenergic response to surgical stimulation, associated to circulatory effects of anesthetic agents, of tracheal intubation, of positive pressure ventilation, of blood losses, of fluid displacement, of 
surgical positioning and of postoperative pain, is one of those factors. Postoperative death-related events include acute myocardial infarction (AMI), arrhythmias and multiple organs failure secondary to low cardiac output ${ }^{2}$.

Vaginal hysterectomy, described by Heaney in 1934, has some advantages over the abdominal procedure. Intestinal loops are less manipulated and there is less postoperative pain, in addition to shorter hospitalization and recovery period $^{3-6}$. However, this procedure requires patient's positioning in exaggerated lithotomy, which may be related to significant hemodynamic changes ${ }^{7}$. This report aimed at describing the case of a patient with dilated cardiomyopathy submitted to vaginal hysterectomy associated to invasive hemodynamic monitoring and neuraxial block.

\section{CASE REPORT}

Black, obese patient (BMI: 32), 33 years old, with history of abnormal uterine bleeding affecting hemoglobin level (hemoglobin: $5.7 \mathrm{~g} / \mathrm{dL}$ ), not improving with clinical treatment. Pelvic evaluation showed good vaginal amplitude and increased uterus with preserved mobility. Ultrasound evidenced myomas of approximately $420 \mathrm{~cm}^{3}$.

Patient had unexplained thrombophilia with history of two strokes without sequelae and one extensive AMI eight years ago evolving to cardiogenic shock, and one AMI without upper unlevelling of ST segment one year ago. Echocardiogram showed increased left atrium $(50 \mathrm{~mm})$ and ventricle $(67 \mathrm{~mm})$, increased right chambers, posterior and inferior septal wall and apex akinesia, marked anterior and lateral walls hypokinesia, with estimated ejection fraction of 0.28 . Cineangiocoronariography showed coronary circulation with right dominance, coronary arteries without significant injuries and severe diffuse left ventricle hypokinesia.

Due to lack of atherosclerosis, coronary syndrome was attributed to thrombotic phenomena secondary to thrombophilia. Patient was under enalapril (5 mg every 12 hours), spironolactone (25 mg/day), furosemide (40 mg/day), carvedilol ( $25 \mathrm{mg}$ every 12 hours) and warfarin ( $7 \mathrm{mg} / \mathrm{day}$ ).

Warfarin was withdrawn four days before surgery and $80 \mathrm{mg}$ enoxaparin were administered every 12 hours. Last enoxaparin dose was administered 24 hours before surgery, with RNI and APTT within reference values the day before surgery. Enoxaparin was reintroduced 12 hours after surgery and warfarin the day after. Chronic medications were maintained. Patient was premedicated with $2 \mathrm{mg}$ lorazepam at 10 p.m. the day before surgery and at 6 a.m. of surgery day with fast as from midnight.

Patient was noninvasively monitored in the operating room and, after sedation, left radial artery catheter was inserted and pulmonary artery catheter (PAC) was inserted through the right subclavian vein with continuous cardiac output monitoring (Baxter-Edwards ${ }^{\circledR}$ ). Baseline hemodynamic parameters were measured after monitoring. Then, spinal anesthesia was performed with $15 \mathrm{mg}$ of $0.5 \%$ hyperbaric bupivacaine and $200 \mu \mathrm{g}$ morphine. Approximately $15 \mathrm{~min}-$ utes after lumbar puncture with sensory level in $T_{8}$, patient was placed in exaggerated lithotomy position for vaginal hysterectomy by the Heaney technique with bilateral salpingectomy. During surgery, $4 \mathrm{mg}$ ondansetron and $8 \mathrm{mg}$ dexametasone were administered to prevent postoperative nausea and vomiting, in addition to $1200 \mathrm{~mL}$ of $0.9 \%$ saline solution.

Hemodynamic parameters were recorded before blockade, 20 minutes after blockade, after beginning dobutamine by infusion pump, 5 minutes after removing patient from the exaggerated lithotomy position and 20 minutes after surgery completion (Table I). Intraoperative intercurrences were post-positioning decreased cardiac index and increased right chambers pressure. Surgery lasted 35 minutes and anesthetic procedure $1 \mathrm{~h} 50 \mathrm{~m}$; at surgery completion patient was referred to the ICU where she remained for 24 hours. Patient was discharged in the third postoperative day.

Table I - Perioperative Hemodynamic Parameters

\begin{tabular}{|c|c|c|c|c|c|}
\hline & $\begin{array}{l}\text { Before Spinal } \\
\text { Anesthesia }\end{array}$ & $\begin{array}{l}5 \text { Minutes after } \\
\text { Positioning } \\
\end{array}$ & $\begin{array}{c}\text { With } \\
\text { Dobutamine }\end{array}$ & $\begin{array}{l}\text { After Removal from } \\
\text { Forced Lithotomy }\end{array}$ & $\begin{array}{l}20 \text { Minutes after Removal } \\
\text { from Position }\end{array}$ \\
\hline MBP & $85 \mathrm{mmHg}$ & $94 \mathrm{mmHg}$ & $110 \mathrm{mmHg}$ & $104 \mathrm{mmHg}$ & $104 \mathrm{mmHg}$ \\
\hline CVP & $3 \mathrm{mmHg}$ & $9 \mathrm{mmHg}$ & $10 \mathrm{mmHg}$ & $8 \mathrm{mmHg}$ & $8 \mathrm{mmHg}$ \\
\hline PAPm & $31 \mathrm{mmHg}$ & $28 \mathrm{mmHg}$ & $40 \mathrm{mmHg}$ & $28 \mathrm{mmHg}$ & $36 \mathrm{mmHg}$ \\
\hline PWP & $15 \mathrm{mmHg}$ & $22 \mathrm{mmHg}$ & $28 \mathrm{mmHg}$ & $15 \mathrm{mmHg}$ & $23 \mathrm{mmHg}$ \\
\hline $\mathrm{HR}$ & 83 bpm & 75 bpm & 70 bpm & 70 bpm & 75 bpm \\
\hline $\mathrm{CO}$ & 5.4 & 4.8 & 6 & 6.1 & 5.6 \\
\hline $\mathrm{Cl}$ & 2.7 & 2.4 & 3 & 3.1 & 2.8 \\
\hline SVRI & 2423 & 2845 & 2654 & 2499 & 2718 \\
\hline PVRI & 473 & 201 & 319 & 338 & 368 \\
\hline SVI & 33 & 32 & 43 & 44 & 38 \\
\hline
\end{tabular}

MBP - mean blood pressure ( $\mathrm{mmHg}$ ), CVP - central venous pressure (mmHg), PAPm - mean pulmonary artery pressure (mmHg), PWP - pulmonary wedge pressure $(\mathrm{mmHg})$, HR - heart rate, CO - cardiac output (L/min), CI - cardiac index, SVRI - systemic vascular resistance index, PVRI - pulmonary vascular resistance index, SVI - systolic volume index 


\section{DISCUSSION}

Preoperative evaluation of cardiac patients should take into consideration their clinical factors and functional capacity, in addition to surgery-related factors.

The objective of this preoperative cardiac risk stratification is to introduce changes in perioperative management to decrease morbidity/mortality. Surgery may be postponed due to unstable symptoms, there may be specific preoperative preparation (pharmacological, volemic or mechanic stabilization), preoperative interventions may be needed (valvoplasty, pacemaker, angioplasty, myocardial revascularization), and postoperative period in ICUs should be defined ${ }^{8}$. Our patient had stable cardiovascular parameters in pre-operative evaluation and was classified as NYHA functional class III, thus included among more severe risk predictors and to be submitted to intermediate risk procedure.

Invasive monitoring was achieved with IAP and PAC (intra-arterial pressure and pulmonary artery catheter) being patient referred to postoperative intensive care unit. This monitoring allowed intraoperative volemic handling, the administration of dobutamine and the diagnosis of position-related hemodynamic effects. Baseline values were CVP $=3 \mathrm{mmHg}$ and PWP $=16 \mathrm{mmHg}$. The administration of $500 \mathrm{~mL}$ of $0.9 \%$ saline as volemic test has increased CVP to 6 and PWP to 22 $\mathrm{mmHg}$, showing severe left ventricular failure due to diastolic restriction.

Vaginal hysterectomy was chosen due to its shorter surgical length and lower postoperative inflammatory response as compared to the abdominal procedure ${ }^{4-6}$. Even for patients with uterus weighing above 250 grams, the vaginal procedure means shorter hospitalization time ${ }^{1,9}$ versus 3.7 days; $p$ $<0.001)$ and postoperative ileum $(1.1 \%$ versus $10.5 \%$; $p=$ 0.006 ) as compared to the abdominal procedure, without increasing morbidity ${ }^{6}$.

Exaggerated head-down lithotomy was the surgical position of choice. This position combines the worst characteristics of both positions alone. Exaggerated lithotomy compresses abdominal organs with subsequent diaphragm raising, pulmonary compression (leading to increased intra-thoracic pressures and changes in ventilation-perfusion rate) and decreased venous return.

Head-down position increases myocardial oxygen consumption, increases intracranial pressure and promotes reflex vasodilation ${ }^{7}$. Abdominal compartment syndrome, rhabdomyolysis, neuropathies, pubic symphyseal separation, lower limbs ischemia, ulcers, pressure alopecia and hypotension are risks associated to this position ${ }^{7,9,10}$. Sometimes, due to poor tolerance to the position, ventilation control is needed. In our case, decreased cardiac index, increased systemic vascular resistance, increased pulmonary artery and wedge pressures were diagnosed after exaggerated lithotomy.

Dobutamine $\left(5 \mu \mathrm{g} \cdot \mathrm{kg}^{-1} \cdot \mathrm{min}^{-1}\right)$ was started to improve cardiac index and decrease pulmonary artery and wedge pressures, and systemic vascular resistance. The noxious effect of posi- tion in a patient with severe cardiac impairment was proven by improved hemodynamic parameters after returning her to the supine position with decreased dobutamine infusion and further withdrawal.

The combination of adequate anesthetic-surgical techniques allows further safety and lower morbidity/mortality in critical patients. Vaginal hysterectomy is safe for severe cardiac disease patients, however it promotes exaggerated lithotomy-related hemodynamic changes which may be poorly tolerated and promote severe cardiovascular complications.

\section{REFERÊNCIAS - REFERENCES}

01. Morgan GE, Mikhail MS - Anestesia para Pacientes com Doença Cardiovascular, em: Morgan GE, Mikhail MS - Anestesiologia Clínica. $2^{\mathrm{a}}$ Ed, Rio de Janeiro, Revinter, 2003;325-359.

02. Koh SH, Rogers J - Anaesthesia for patients with cardiac disease undergoing non-cardiac surgery. Update in Anesthesia, 2002;14:11-16.

03. Heaney NS - Vaginal hysterectomy: its indications and technique. Am J Surg, 1940;48:284-288.

04. Silva-Filho AL, Reis FM, Noviello MB et al - Factors influencing the operative time and complications of vaginal hysterectomy of a nonprolapsed uterus. J Pel Med Surg, 2004;10:257-262.

05. Harmanli OH, Gentzler CK, Byun S et al - A comparison of abdominal and vaginal hysterectomy for the large uterus. Int $\mathrm{J}$ Gynaecol Obstet, 2004;87:19-23.

06. Ribeiro SC, Ribeiro RM, Santos NC et al - A randomized study of total abdominal, vaginal and laparoscopic hysterectomy. Int J Gynaecol Obstet, 2003;83:37-43.

07. Warner MA, Martin JT - Patient Positioning, em: Barash PG, Cullen BF, Stoelting, RK - Clinical Anesthesia. $4^{\text {th }}$ Ed, New York, Lippincott Willians \& Willians, 2001; 639-666.

08. Park KW - Preoperative cardiology consultation. Anesthesiology, 2003;98:754-762.

09. Gherman RB, Ouzounian JG, Incerpi MH et al - Symphyseal separation and transient femoral neuropathy associated with McRoberts maneuver. Am J Obstet Gynecol, 1998;178: 609-610.

10. Bocca G, van Moorselaar JA, Feitz WF et al - Compartment sydrome, rhabdomyolysis and risk of acute renal failure as complications of the lithotomy position. J Nephrol, 2002;15: 183-185.

\section{RESUMEN}

Bessa Jr RC, Silva Filho AL, Maia PV, Quites LO, Triginelli AS Repercusiones Hemodinámicas de la Posición de Litotomía Exagerada para Histerectomía Vaginal en una Paciente con Cardiopatía. Relato del Caso.

JUSTIFICATIVA Y OBJETIVOS: La técnica de histerectomía vaginal permite menor tiempo operatorio y el uso de bloqueo espinal, con los beneficios en la analgesia post-operatoria y en la menor respuesta sistémica frente al procedimiento quirúrgico. El objetivo de este relato es describir las alteraciones hemodinámicas secundarias al posicionamiento en litotomía exagerada en una paciente con cardiopatía. 
RELATO DEL CASO: Paciente de 33 años, GOPOAO, con historia de sangrado uterino anormal y anemia. La ultra-sonografía evidenciaba útero miomatoso con volumen estimado de $420 \mathrm{~cm}^{3}$. Portadora de miocardiopatía dilatada, refería dos accidentes vasculares isquémicos y dos infartos agudos de miocardio en el pasado. Fue monitorizada con presión arterial invasiva y catéter en la arteria pulmonar para medir el gasto cardíaco en forma continua. Fue realizada raquianestesia con bupivacaína hiperbárica y morfina. La paciente fue posicionada en litotomía exagerada para realizar histerectomía total con la técnica de Heaney y salpingectomía bilateral. Durante el intra-operatorio, luego del posicionamiento, presentó disminución del índice cardíaco y aumento de presión en las cámaras derechas, que requirió tratamiento con dobutamina.

CONCLUSIONES: La posición de litotomía exagerada puede ser causa de alteraciones hemodinámicas que deben ser consideradas al seleccionar la técnica quirúrgica. 